\title{
LA OBSERVACIÓN, UN MÉTODO PARA EL ESTUDIO DE LA REALIDAD
}

\section{OBSERVATION, A METODY FORING THE ESTUDY OF REALITY}

Guillermo Campos y Covarrubias

Lic. en Antropología Social. Maestro en Administración de Negocios, Maestro en administración pública, Maestro en Sociología, Doctor en

Administración Pública y Doctor en Ciencias de la Educación. Profesor de carrera de tiempo completo en la UNAM, miembro del

SNI nivel I, así como miembro del COMIES. Sus líneas de investigación son: migración, educación, salud y cultura. Ha publicado varios libros como resultado de la investigación. ccg482007@gmail.com

Nallely Emma Lule Martínez

Lic. En Trabajo Social por la Escuela Nacional de Trabajo Social. Actualmente estudiante de Maestría en Salud Pública por el Instituto Nacional de Salud Pública. Ha sido docente del Colegio de Posgrados de la Ciudad de México, cuenta con diversas publicaciones y ha participado en varios proyectos y encuentros de investigación. Sus líneas de trabajo son: Educación, Salud, Mercado Laboral, Migración, Identidad y Cultura. E-mail: gc_nelm_gl@hotmail.com

\section{Resumen}

El propósito de este trabajo es encaminar a los estudiantes hacia la utilización y comprensión de una de las técnicas de investigación más utilizadas, pero a su vez una de las más complejas; la observación. La observación por sí misma representa una de las formas más sistematizadas y lógicas para el registro visual y verificable de lo que se pretende conocer, consiste en utilizar los sentidos ya sea para describir, analizar, o explicar desde una perspectiva científica, válida y confiable algún hecho, objeto o fenómeno desde una forma 
participante, no participante, estructurada o no estructurada; de esta forma se plantea la necesidad de que el observador cuente con habilidades y destrezas que le permitan desarrollar este proceso con calidad.

Palabras clave: Técnica, investigación social, validez, objetividad, sistemática.

\begin{abstract}
The purpose of this paper is to connect students to the use and understanding of one of the most widely used research techniques, but also one of the most complex and the observation. The observation itself is one of the most systematic and logical for the visualand verifiable record what we want to know is to use either way to describe, analyze, or explain from a scientific perspective, a valid and reliable Indeed, object or phenomenon from a participant is not participating, structured or unstructured, in this way raises theneed for the observer count skills that allow them to develop this process with quality.
\end{abstract}

Keywords: Technical, social research, validity, objectivity, systematic.

\title{
Introducción
}

El presente artículo es producto de años de trabajo dentro de la docencia y la investigación social; es decir, de la experiencia empírica adquirida durante toda la vida educacional, es por ello que nos darnos a la tarea de decir que la observación es uno de los problemas comúnmente distorsionado en el medio, puesto que limita y transforma la relación persona-objeto-razón; en donde la problemática enseñanza-aprendizaje se encuentra inmersa en un constante desequilibrio que trae como consecuencia problemas en el desarrollo y crecimiento de las disciplinas.

En este artículo se aborda una de las técnicas de las que más se vale la investigación científica en general y en particular la investigación social, la observación; ésta es una actividad históricamente inherente al hombre, a través de la cual éste llega a 
identificar, saber y conocer su mundo, su vida natural o social; entiendo al ser humano como un ente biopsicosocial que al formar paradigmas teórico-metodológicos da sentido a la ciencia de la cual se vale la humanidad para describir, explicar, analizar o predecir su realidad y su entorno.

La observación no es una actividad sólo practicada por el hombre de ciencia, sino que ésta, es una acción cotidiana de los sujetos que al no ser rígida toma diversos matices de acuerdo al interés de los hombres y a la forma en cómo se utiliza.

\section{La observación y ciencia}

En el amplio campo de la investigación la observación puede ser entendida por algunos como un método; para otros es una técnica; y aun cuando existen puntos de contacto entre método y técnica, existe una diferencia esencial; al primero lo determina en gran medida el área de estudio al que corresponde la investigación, mientras que la segunda es aplicable independientemente del área de estudio; en este sentido y para fines de este documento definiremos a la observación como una técnica que mediante la aplicación de ciertos recursos permite la organización, coherencia y economía de los esfuerzos realizados durante el desarrollo de una investigación; de esta forma, esta técnica tendrá una organización y una coherencia dependiente al método utilizado.

En otras palabras, la observación es la forma más sistematizada y lógica para el registro visual y verificable de lo que se pretende conocer; es decir, es captar de la manera más objetiva posible, lo que ocurre en el mundo real, ya sea para describirlo, analizarlo o explicarlo desde una perspectiva científica; a diferencia de lo que ocurre en el mundo empírico, en el cual el hombre en común utiliza el dato o la información observada de manera práctica para resolver problemas o satisfacer sus necesidades.

De esta forma toda observación, al igual que otras técnicas, métodos o instrumentos para consignar información; requiere de un sujeto que investiga y un objeto a investigar, tener claros los objetivos que persigue y focalizar la unidad de observación. 
Al respecto Bunge (2007) señala que, la observación es el procedimiento empírico elemental de la ciencia que tiene como objeto de estudio uno o varios hechos, objetos o fenómenos de la realidad actual; por lo que en el caso de las ciencias naturales, cualquier dato observado será considerado como algo factual, verdadero o contundente; a diferencia, dentro de las ciencias sociales, el dato será el resultado que se obtiene del proceso entre los sujetos y sus relaciones por lo que no es tan factual y pudiera ser subjetivo.

En términos filosóficos podemos señalar que la observación es un proceso por el cual se filtra la información sensorial a través del proceso que sigue el pensamiento (idea, juicio, razón) del cual se vale el hombre para construir su mundo. Cuando se habla de procesos en la observación, es necesario entender que se trata de secuencias lógicas e intencionadas que tienen determinada temporalidad en un escenario específico. Un proceso puede durar días, semanas o quizá meses, ya que depende del alcance, experiencia y propósitos del investigador ante el fenómeno, objeto o hecho en estudio.

De esta manera el hecho en estudio debe ser considerado como un acontecimiento de la realidad observada, el cual contempla elementos abstractos (teoría) y elementos prácticos (empíricos) para el observador. Por lo tanto, un hecho no es sólo un producto de cualquier cosa o situación observada, sino es parte de una realidad y representa un objeto y/o una circunstancia conocida o factible de conocer.

El objeto de observación se constituye por todo aquel hecho o fenómeno en el que se puede distinguir la esencia a observar: cualidades y características, que le dan sentido e identidad a éste; y por consecuencia le dan: forma, prestancia y claridad a la observación. El objeto de estudio puede o no ser parte del investigador que observa; ya que desde una visión positivista, empirista y materialista, al objeto de estudio se le analiza fuera del consciente del sujeto (observador); es decir, la opinión de quien observa está determinada por el fenómeno o hecho que se estudia; mientras que desde el humanismo y la fenomenología, el observador 
es parte de la realidad, por lo cual la interpreta a partir de su intervención directa en el objeto de estudio.

Por otra parte, el fenómeno se entiende como todo suceso natural o social que existe independientemente del observador; el fenómeno, es algo perceptible que se presenta como realidad objetiva en un espacio y tiempo, fiable de ser visto por sus atributos tangibles, por lo tanto, éste puede causar confusión al tratar de conocerle, de ahí que se diga que observar, no sólo es ver, sino analizar, siendo esto ultimo el papel que debe jugar el investigador al momento de hacer la observación.

Por lo tanto, la observación debe realizarse con el uso de todos los sentidos, de tal modo que le permitan al investigador configurar la realidad de una manera empírica y teórica para su comprensión; en donde la sospecha, la intuición, las corazonadas, son impulsos que no deben ser determinantes para el investigador y trabajos de carácter científico.

Cabe mencionar, que la observación requiere de atributos para su aplicación, así como también el desarrollo de habilidades previas que establezcan las condiciones de manera tal, que los hechos observables se realicen en la forma más natural; es decir, sin que exista la intervención de juicios previos y de esta forma dar pie a prever la conducta que asumirá el investigador ante el fenómeno a observar; o sea, tomar en cuenta hasta donde sea posible, todos los elementos que puedan intervenir de forma directa o indirecta en el proceso de la observación. En otras palabras, el fenómeno se debe observar bajo cierto control en donde se deben tomar en cuenta cuestiones como ¿Qué se quiere saber? ¿Para qué se quiere saber? ¿En cuánto tiempo lo quiero saber? y así evaluar los hechos durante la observación.

\section{La observación como base de la investigación}

Dentro de la investigación social al igual que en otras disciplinas de las ciencias, se utilizan métodos y técnicas de recolección de información que de una u otra forma permiten reconocer aspectos propios de la realidad así como las formas cuantitativas y cualitativas.

Dichos aspectos permiten analizar, describir, inferir, interpretar, 
aprobar o rechazar la formulación de teorías sobre los procesos; así como diagnósticos de realidades y modelos de intervención, todos ellos fundamentados en datos obtenidos de la observación.

Los observadores del campo de la investigación deben estar conscientes de la existencia de las diferentes posturas; por esta razón es necesario definir la ruta a seguir al emplear la observación, puesto que de aquí depende el cómo nos acerquemos a la realidad y se irá configurando el objeto de estudio atribuyéndole elementos sólidos que permitan la interpretación y comprensión del objeto en cuestión.

\section{La observación como técnica ¿para qué?}

Desde el punto de vista de Ander-Egg (2003) la observación presenta dos acepciones; la primera se relaciona con la técnica de investigación, la cual participa en los procedimientos para la obtención de información del objeto de estudio derivado de las ciencias humanas, empleando los sentidos con determinada lógica relacional de los hechos; y la segunda, como instrumento de investigación el cual se emplea de manera sistemática para obtener información a través de los principios del método científico buscando la validez y confiabilidad de los datos obtenidos.

Para nosotros este es el punto de partida para decir que la observación es un procedimiento que ayuda a la recolección de datos e información y que consiste en utilizar los sentidos y la lógica para tener un análisis más detallado en cuanto a los hechos y las realidades que conforman el objeto de estudio; es decir, se refiere regularmente a las acciones cotidianas que arrojan los datos para el observador.

Por lo tanto, para los fines de este documento se define la observación como una técnica por medio de la cual se crea una vinculación concreta y constante entre el investigador y el hecho o fenómeno, y que tiende a ceñirse a la lógica de las formas, procedimientos, relaciones e interrelaciones que se suscitan dentro de la realidad estudiada. 
De esta manera la observación, al ser empleada para la recopilación de datos por parte del investigador, debe tomar en cuenta:

1. Que debe ser planteada con el fin de garantizar validez y confiabilidad.

2. Que se requieren habilidades y destrezas por parte de quien observa para que ésta sea realizada con calidad y sentido sistemático en el registro de los datos.

3. Que debe sobrepasar lo analógico de lo analítico.

Por su parte Selltiz (citado por Hernández; 2006, p.229) al referirse a la observación, recomienda que para que ésta se convierta en una técnica como tal, debe cumplir con cuatro condiciones:

1. Debe servir a un objeto formulado de investigación.

2. Debe de ser planificada sistemáticamente.

3. Debe estar controlada y relacionada con proposiciones generales.

4. Debe ser sujeta a comprobaciones y controles de validez y fiabilidad.

De acuerdo a lo anterior, podemos asumir que la observación:

1. Tiene la característica de seguir normas, reglas y procedimientos.

2. Permite a los sujetos y objetos establecer relaciones de manera directa.

3. Esas relaciones sirven para acumular, sistematizar y registrar los datos obtenidos.

\section{Clasificación de la observación}

La observación de los fenómenos o hechos regularmente depende de la postura que adopta el investigador o bien, suponen habitualmente la toma de decisiones deliberadas del investigador o el equipo de investigación que focaliza la atención a uno o varios aspectos o categorías significativas de lo que se estudia en una situación concreta representada en espacio y tiempo determinados; es por ello que se hace hincapié en que el observador debe ser ajeno a cuestiones externas del objeto de estudio y así evitar intervenciones que arrojen resultados no verdaderos, para poder alejarse de cuestiones que compliquen la observación es importante tomar en cuenta bajo qué criterios y modalidades resulta más 
conveniente o apropiado desplegar la observación; por lo tanto, resulta primordial tener en cuenta; por un lado, que la postura epistemológica que el investigador tomará, así como la metodología que se implementará dependen de la naturaleza del objeto que se investigue; y por otra parte que, el objeto de estudio responderá a los criterios, necesidades y experiencias del investigador.

Con base en lo anterior, la observación se puede clasificarse dentro de las siguientes modalidades:

1. Observación de laboratorio: Se desarrolla es espacios que permiten la reproducción de los hechos y se tiene el control de lo que puede acontecer en un estado real para su generalización.

2. Observación no participante: Se trata de una observación realizada por agentes externos que no tienen intervención alguna dentro de los hechos; por lo tanto no existe una relación con los sujetos del escenario; tan sólo se es espectador de lo que ocurre, y el investigador se limita a tomar nota de lo que sucede para conseguir sus fines.

3. Observación participante: El investigador se involucra dentro de los procesos de quienes observa, y éste es plenamente aceptado, por lo tanto, se estima que lo observado no se ve afectado por la acción del observador. Padua (1987)

4. Observación no estructurada: En ella se trata de observar sin tener en cuenta categorías o indicadores que guíen el proceso; careciendo de control temporal y llevando a cabo registros libres y globales de los acontecimientos. Puede también ser llamada observación no sistemática u ocasional, ya que registra todo aquello que se observa y se realiza cuando no se conoce suficiente del objeto de estudio, y se puede llegar a emplear para hacer estudios piloto; aunque por otra parte, al hacer uso de esta técnica se corre el riesgo de registrar procesos aislados y de poca funcionalidad o relevancia.

5. Observación estructurada: Se refiere a la observación metódica que es apoyada por los instrumentos como la guía de observación y el diario de campo mediante la utilización de categorías previamente codificadas y así poder obtener información controlada, clasificada y sistemática. 
Como en todos los procesos, en la observación, es de suma importancia que se tenga presente y en claro el tiempo de empleo de los criterios metodológicos a implementar, a efecto de darle forma e impulso a la manera de proceder ante esa realidad observada; es decir, resulta imperante el reconocimiento de la metodología más conveniente para el alcance de los propósitos en el tiempo estimado y así para evitar la aparición de variables extrañas en la investigación; por lo que es importante tener en cuenta:

1. Qué o quiénes se observarán para así esclarecer el objeto de estudio y los fines específicos de esa acción.

2. Determinar cuál es la fundamentación con que se rige la observación, y así establecer la forma de realizarla y los alcances que se estiman alcanzar con ella.

3. Diseñar las guías de observación que auxilien a sistematizar el proceso; así como la duración y frecuencia de los registros; la funcionalidad de estas guías estará sujeta al tipo de observación que se emplee ya que son inexistentes dentro de la observación no estructurada.

4. Realizar registros de observación con base en las guías, según sea el caso, para valorar si los tiempos, las manifestaciones, 0 las actuaciones de los sujetos 0 fenómenos que se registran en ellas son los necesarios para cubrir con los objetivos de la investigación.

5. Seleccionar los procesos que darán cuenta al análisis de lo observado.

Desde el punto de la investigación social, es importante tener presente los criterios antes referidos para así poder seleccionar con precisión la o las modalidades de observación a emplear según sea el caso, ya que es a través de ellas como será posible ser congruente con la naturaleza del objeto de estudio, el enfoque metodológico que orienta el trabajo de campo y, la interpretación de la información que conlleve a resultados reflexivos en apego a la realidad que se investiga.

\section{Las categorías de análisis en la observación}


Las categorías en la investigación incluyen definiciones y conceptos que contribuyen a la construcción de la teoría; del objeto de estudio que se observa, también permiten dar vida y sentido al contexto ya que focalizan la atención epistémica y teórica del observador; dicho de otra forma, las categorías son clasificaciones básicas de las conceptualizaciones referidas del objeto de estudio, y describen a las clases de sujetos, contextos u objetos de los que puede decirse algo específicamente dentro de su situación real.

Desde esa lógica, dentro de las categorías de análisis se trata de ir más allá de la simple mirada del hecho o fenómeno manifiesto, puesto que el propósito de la elaboración de categorías es alcanzar un grado de interpretación hermenéutico/dialéctico; es decir, obtener la comprensión, interpretación y aplicación de la literatura ante el objeto de estudio existente, por consiguiente, conlleva a romper con la visión empírica de lo que es observado.

Es así como desde la perspectiva de Rodríguez (1999), se resalta que en las investigaciones de corte cualitativo existe una gran cantidad de información, por lo que se hace necesario categorizarlos para facilitar su análisis y poder responder a los objetivos que pueden ser cambiantes en tanto se va obte-niendo la información. En ese sentido, las categorías pueden desarro-llarse utilizando una palabra de una idea que sea similar en otras ideas o creando un nombre con base en un criterio unificador e integrador, logrando que al final del proceso todas las ideas estén incluidas en alguna categoría. Al construir las categorías no debe existir la intervención de interpretaciones previas que se asocien a prejuicios, por lo tanto, siempre se debe respetar la información obtenida del proceso de la observación.

Las categorías de análisis se manifiestan en el pensamiento crítico de lo que se observa, las cuales cobran sentido cuando se vinculan con la realidad del objeto de estudio. Su construcción responde a cuestiones dialécticas, metodológicas y a la perspectiva disciplinaria con que el investigador cuenta.

A decir de Rodríguez, (1999) las categorías en la observación conllevan hacia un modelo teórico definido con precisión y claridad que guía al observador para recoger hechos directos; por lo tanto, 
permiten construir guías orientadoras que acerquen al investigador a la realidad.

Por ejemplo: En un estudio sobre "La enseñanza de las competencias comunicativas" la categoría de análisis podría ser la estrategia didáctica que se emplea; de esta manera lo que el investigador debe observar:

1. La intencionalidad de las estrategias.

2. Las características de las estrategias.

3. El dominio que tiene el docente de las estrategias; (tan sólo por mencionar algunos indicadores).

De esta menara, queda claro que con las categorías es posible emprender un proceso de observación orientado, en el que se evita observar sin sentido y el riesgo de obtener información con poco valor y utilidad; así, tanto las categorías como los indicadores, buscan vincular la teoría con la realidad.

\section{Guía de observación, instrumento central}

La guía de observación es el instrumento que permite al observador situarse de manera sistemática en aquello que realmente es objeto de estudio para la investigación; también es el medio que conduce la recolección y obtención de datos e información de un hecho o fenómeno.

Tamayo (2004, p.172) define a la guía de observación como:

Un formato en el cual se pueden recolectar los datos en sistemática y se pueden registrar en forma uniforme, su utilidad consiste en ofrecer una revisión clara y objetiva de los hechos, agrupa los datos según necesidades específicas, se hace respondiendo a la estructura de las variables o elementos del problema.

El proceso a seguir para realizar una guía de observación es propiamente, saber qué se quiere conocer, focalizándolo en el fenómeno o problema, sin perder de vista que es fundamental que el instrumento tenga validez y confiabilidad. 
La validez se refiere a la elaboración de un instrumento que sea capaz de medir lo que se desea que mida; y que a su vez esta medición sea posible de realizarse; en otras palabras, que refleje lo que se tiene como propósito y realice una medición objetiva y veraz. Por otra parte, la confiabilidad hace alusión a la precisión y estabilidad que el instrumento tenga en distintos escenarios o momentos de estudio; es decir, que sea capaz de soportar el análisis en cualquier momento de la investigación sin perder su validez.

Una vez dicho lo anterior, se tiene que para elaborar el instrumento se deben cumplir dos faces:

Primera fase:

1. Contar con el objeto de investigación bien planteado.

2. Tener claridad en los objetivos de la investigación.

3. Presentar las categorías del objeto de estudio.

4. Seleccionar con precisión los indicadores a observar.

5. Estructurar el diseño de la guía de observación.

6. Así como definir le encuadre cualitativo o cuantitativo del instrumento.

Segunda fase:

1. Valorar las condiciones para llevar la observación.

2. Organizar la temporalidad de la observación.

3. Sistematizar los lapsos específicos para los registros.

4. Diseñar símbolos representativos de las acciones.

5. Contar con los medios para concretar lo observado: Formatos de registro.

6. Considerar la actitud, vestuario y postura de su personalidad al observar.

Para elaborar la guía de observación se ha de diseñar el encuadre de la observación, que debe por lo menos incluir los siguientes aspectos:

1. Datos y características de los sujetos a evaluar.

2. Propósitos de la observación o de las observaciones a realizar.

3. Temporalidad de la observación. 
4. Lapsos o rangos de los momentos observables en apego a las categorías.

\section{Ventajas y desventajas de la observación}

La observación en la investigación educativa tiene sus alcances y limitaciones a la vez, los primeros se asocian a las ventajas y los otros a las desventajas que ésta tiene:

Ventajas:

1. Se observa de manera natural a los acontecimientos.

2. Describe los hechos de manera exacta.

3. Obtiene elementos significativos desde una perspectiva específica al considerar categorías e indicadores.

4. Representa un bajo costo monetario y material para el investigador.

Desventajas:

1. Puede existir una falta de dominio de las categorías o indicadores a observar.

2. Existen variables difíciles de observar, lo que puede causar confusión.

3. Se corre el riesgo de sesgar lo observado.

4. Se pueden emplear juicios erróneos al no vincular de manera adecuada las categorías o indicadores con la realidad.

5. No se pueden generalizar los resultados de la observación porque cada sujeto, grupo y contexto suele tener características específicas.

\section{Consideraciones finales}

A lo largo de nuestra experiencia dentro del campo de la investigación y la docencia hemos identificado la preocupación que representa para los estudiantes enfrentarse a los retos y desafíos de la investigación y más allá definir las técnicas que les permitan finalizar los procesos necesarios o complementarios de su formación.

De esta manera se concluye que la utilización de la técnica de observación es una posibilidad que no se debe dejar de lado, 
considerando a la guía de observación como un aliado que da cuenta de la ruta, los momentos y los espacios en que se puede observar a la realidad; ya sea para:

1. Tener un primer acercamiento de lo desconocido para que conlleve a diagnósticos, o

2. Como una visualización a lo poco conocido a través de estudios exploratorios.

3. Indagar una realidad manifiesta que conlleve a una explicación o bien.

4. Para interpretar lo que acontece en un contexto para su análisis, descripción, crítica o comprensión.

\section{Agradecimientos}

Este documento es resultado de la revisión bibliográfica del grupo de investigación del proyecto PAPIIT IN302810 "Las representaciones sociales y el mercado laboral de la enfermería profesional en México" de la Escuela Nacional de Enfermería y Obstetricia de la UNAM, durante el año 2010; en donde actividades como el ficheo y la sistematización de datos e información han llevado al desarrollo de material de apoyo no sólo para los participantes en el proyecto, sino un material de consulta para todo el interesado en esta actividad.

\section{FueNTES DE CONSULTA}

- ANDER-EGG, E. (2003). Métodos y Técnicas de Investigación Social. Buenos Aires: Lumen Hvmanitas.

- ANDER-EGG, E. (1971). Introducción a las Técnicas de Investigación Social. Buenos Aires: Lumen Hvmanitas.

- BUNGE, M. (2007). La investigación científica. México, Siglo XXI.

- HERNÁNDEZ, S. FERNÁNDEZ C. y BAPTISTA L. (2006). Metodología de la investigación. México: Mc Graw Hill.

- PADUA, J. et. al. (1987) Técnicas de investigación aplicadas a las ciencias sociales. México: COLMEX/FCE. 
- RODRígueZ, G, GÓMEZ, J, FLORES G. y GARCÉS E. (1999). Metodología de Investigación Cualitativa. España: Algibe.

- SORIANO, R. (2006). Guía para realizar investigaciones sociales. México: UNAM.

- TAMAYO, M. (2004). El proceso de la investigación científica. México: Limusa 